\section{Viscosupplementation for the treatment of osteoarthritis. The contribution of EUROVISCO group}

\author{
Thierry Conrozier (D), Raghu Raman, Xavier Chevalier, Yves Henrotin, Jordi Monfort, \\ Demirhan Diraçoglù, Hervé Bard, Dominique Baron, Jörg Jerosch, Pascal Richette \\ and Alberto Migliore
}

\begin{abstract}
Viscosupplementation (VS) is a symptomatic treatment for knee and other joint osteoarthritis (OA). Despite a long history of use, conflicting opinions remain on the best clinical indications and the most appropriate patients to be treated with intra-articular hyaluronic acid (IA-HA), the optimal dosing regimen and the modalities of retreatment. A multidisciplinary committee of European experts on OA (EUROVISCO) was constituted to formulate recommendations, aimed at helping physicians in the decision-making and the optimal achievement of VS. Before each session members were tasked to collate an exhaustive literature review. Level of evidence and strength of recommendation were based on the level of agreement for each item according to the Delphi method. In 2015, a consensus position was proposed for 24 statements. Among those that obtained a consensual agreement, the working group stressed that VS is effective in mild/moderate knee OA but is not an alternative to surgery in advanced OA, and that dosing regimen must be supported by controlled trials. In 2018, two decision algorithms for the retreatment with IA-HA in knee OA were published. Among the key recommendations, the experts recommended to re-treat every year patients with high risk of OA progression, even if not symptomatic. In 2020, EUROVISCO published two sets of recommendations for the design of clinical trials on the disease-modifying effect of VS and for optimizing the results of VS. The working group underlined that an accurate analysis of radiological features and symptoms and a careful clinical examination may improve the chances of success of VS, as well as good technique of injection and the use of imaging guidance. Based on the exhaustive analysis of the literature and their own clinical experience, the EUROVISCO experts offer a wide range of recommendations intended to help practitioners, particularly in certain cases where the specific characteristics of the patients make the therapeutic decision difficult.
\end{abstract}

Keywords: viscosupplementation, hyaluronic acid, intra-articular injection, osteoarthritis, knee, hip, recommendations, EUROVISCO

Received: 13 January 2021; revised manuscript accepted: 28 April 2021.

\section{Introduction}

Viscosupplementation (VS), by intra-articular (IA) injection of hyaluronic acid (HA), has been a globally used symptomatic treatment for knee and other joint osteoarthritis (OA) for over a quarter-century. ${ }^{1}$ VS is recommended in the management of symptomatic knee OA, for appropriate patients, by many scholarly societies of rheumatology and orthopaedics, ${ }^{2-6}$ geriatrics ${ }^{7}$ and sport medicine. ${ }^{8}$ These recommendations are based on systematic reviews and meta-analyses that consider VS as an efficient and reliable therapy. ${ }^{9-13}$ When compared with IA corticosteroids (CSs), IA-HA has been shown to have a more long-lasting effect for relieving knee pain (up to 6 months), while IA-CS is more effective on pain relief in the short term (up to 1 month), with similar safety profiles. ${ }^{14-16}$ The good safety of IA-HA is no longer under question. ${ }^{2-8}$ However, few authors underline the lack of adverse event
Ther Adv Musculoskel Dis 2021, Vol. 13: 1-8 DOI: $10.1177 /$ $1759720 \times 211018605$

(c) The Author(s), 2021. Article reuse guidelines: sagepub.com/journalspermissions

Correspondence to: Thierry Conrozier Department of

Rheumatology, Hôpital Nord Franche-Comté, 100 route de Moval, CS 10499

Trévenans, Belfort, 90015, France

thierry.conrozierahnfc.fr

Raghu Raman

Academic Department of Orthopaedics, Hull and East Yorkshire NHS Trust Castle Hill Hospital, Cottingham, UK

Xavier Chevalier

Paris XII University,

UPEC, Department of

Rheumatology, Henri

Mondor Hospital, Creteil,

France

Yves Henrotin

Bone and Cartilage

Research Unit, Universite de Liège, CHU Sart-

Tilman, Liège, Belgium

Jordi Monfort

Servei de Reumatologia, Hospital del Mar, Parc de Salut Mar, Barcelona, Spain

Demirhan Diraçoglù Department of

Physical Medicine and Rehabilitation, Istanbul University and Istanbul

Faculty of Medicine. Istanbul, Turkey

Hervé Bard

Rheumatology Cabinet Medical Vaudoyer, Paris, France

Dominique Baron Centre de réadaptation fonctionnelle de Lannion-Trestel, Trévou-

Tréguignec, France

Jörg Jerosch

Orthopaedic Department Johanna-Etienne-Hospital, Neuss, Germany

Pascal Richette Université Paris Diderot, UFR médicale, Hôpital Lariboisière, Paris, France Alberto Migliore U.O.S. of Rheumatology, Ospedale San Pietro

Fatebenefratelli, Rome, Italy 
synthesis standardization that leads to opposite conclusions about the balance of benefits and harms. ${ }^{17}$

Beyond its clinical effectiveness, when administered in appropriate patients, repeat IA-HA injections have also been suggested to delay the time to arthroplasty. ${ }^{18-20}$ However, despite positive assessment by clinicians and a high level of evidence, some guidelines continue to fail to recommend VS, ${ }^{21-23}$ resulting in a gap between guidelines and practitioners' habits, who continue to use VS in their daily practice. ${ }^{24,25}$ Among the reasons that may explain the remaining doubt about the real effect of VS, Printz et al. underlined the importance of conflicts of interest in the studies' outcomes. ${ }^{26}$

In most countries HA viscosupplements are classified as class III implantable medical devices. The mechanisms by which HA acts on joint tissues are not fully understood and probably very complex. ${ }^{27,28}$ Hence, the longstanding classification of viscosupplements as medical device has been recently called into question by the American Food and Drug Administration, which decided to reclassify HA as a drug, citing evidences of pharmacological effects.

Despite a long history of use, conflicting opinions remain on the best clinical indications, the most appropriate patients to be treated, the optimal dosing regimen and the modalities of retreatment. In 2017 a task force of US clinical experts developed and published Appropriate Use Criteria of VS in knee OA, ${ }^{29}$ aimed to help physicians in the decision making of VS and to help insurance agencies to determine cases where reimbursement could be considered.

In 2014, a task force of European experts on OA was brought together in order to propose a consensual approach on VS in knee and other joint OA. The working group was named EUROVISCO (EUROpean VIScosupplementation COnsensus group). The 11 members come from seven European countries (Belgium, France, Germany, Italy, Spain, Turkey and UK). They constitute a multidisciplinary panel of physicians in the field of OA (seven rheumatologists, two orthopaedic surgeons, two physical therapists). All have experience in both academic medicine and private practice, and expertise in clinical research methodology. Before each session three or four members of the task force were tasked to collate an exhaustive literature review on a topic and to prepare the statements to be discussed. At each working session, one member acts as a chairman: he guides the discussion and plays the role of a moderator to iron out opinion discrepancies among the working group members. For each issue raised, the experts must give a degree of agreement, using a numerical Likert scale, ranging from 'I don't agree at all' to 'I fully agree'. The scores are then pooled to generate a median agreement score for each statement, which allows calculating the strength of recommendation. The number of voters who strongly agree obtains the level of consensus: unanimous if all experts fully agreed with the recommendation, high and moderate respectively if $9 / 10$ and $8 / 7$ experts highly agree.

The working objective of the EUROVISCO task force is to publish simple and clear recommendations aimed to provide clarification and advice to prescribers and users of VS. The first set of recommendations was published in $2015 .^{30} \mathrm{~A}$ consensus position based on an extensive research of the literature and expert opinion was proposed for 24 statements on VS of the knee and other joints. In 2018 the task force proposed two decision algorithms for the retreatment with IA-HA in patients with knee OA. ${ }^{31}$ In 2020, EUROVISCO published a set of recommendations for the design and conduct of clinical trials on the disease-modifying effect of knee $\mathrm{VS}^{32}$ and another set focused on the different ways for optimizing the clinical results of VS. ${ }^{33}$ The aim of the present article is to briefly summarize the main conclusions of these four publications and to discuss the contribution of EUROVISCO group.

\section{Consensus statement on VS with HA \\ for the management of $O A^{30}$}

The aim of this first meeting was to set the record straight on the 'art' of VS and to provide clear and concrete answers to the questions frequently asked by the care-providers. Twenty-four statements exploring the entire field (i.e. effectiveness, safety, injection techniques, imaging guidance) of VS were discussed. Issues addressed included OA of not only the knee but also hip, tapezio-metacarpal joint (TMJ), ankle and shoulder VS. A high level of agreement was achieved for 16 statements or recommendations. In particular the expert achieved unanimous agreement in favour of nine issues. Among them the working group stressed that (1) VS is effective in mild and 
moderate knee OA, (2) VS is a well-tolerated treatment of knee and other joint OA, (3) VS is not an alternative to surgery in advanced hip OA, (4) VS can be proposed in all symptomatic patients, even in those who adequately respond to analgesics or non-steroidal anti inflammatory drugs (NSAIDs) if they prefer receiving IA-HA to taking pain-killers, (5) the dosing regimen must be supported by the results of well conducted controlled trials. Statements that received unanimous or strong level of agreement are summarized in Table 1. A strong level of between-expert agreement was also obtained on the fact that VS may also be helpful in advanced stages of knee $\mathrm{OA}$ and also that when administered at early stages of $\mathrm{OA}$ it might have a chondroprotective effect. Furthermore, the working-group members strongly agreed not to consider HA viscosupplements as a 'single class' due to the wide difference between products. Hence the results of clinical trials of a particular VS cannot be extrapolated to others. They also stressed that a single-injection regimen must be performed with products specifically developed for this irrespective of the joint to be treated. In contrast, the experts could not find a consensus answer regarding the effectiveness of VS in OA of the shoulder and TMJ and on the mandatory use of an imaging guidance when performing VS in non-knee OA. Likewise they did not agree among themselves on the interest to combine HA and CS during the same injection session and on the poorer tolerance of animalderived HA compared with HA of bacterial origin. Statements on VS use that obtained a unanimous or strong level of consensus are given in Table 1.

\section{Decision algorithms for the re-treatment with}

$V S$ in patients suffering from knee $O A^{31}$

In daily clinical practice the re-treatment algorithms vary a lot from country to country and even between physicians. Some re-treat patients when pain returns to baseline level. Others opt for a repeat HA injection systematically, every 6 or 12 months. Although systematic reviews of IA-HA repeat injections showed favourable benefit/risk ratio, ${ }^{34}$ the criteria for re-treatment had never been published before. The goal of the meeting was to examine two frequent clinical situations: re-treatment in patients successfully treated with VS 6 to 12 months ago and in those in whom previous VS failed or caused adverse effects. At the end of the debates the EUROVISCO group proposed two decision algorithms for the management of knee OA patients previously treated - successfully or otherwise - with VS. The expert panel had to give opinion on 88 issues within 18 statements. The first step was to give an accurate definition of 'success' and 'failure' of the treatment. The second step was to determine when and how to re-treat patients successfully treated by a previous VS. The third step was to determine when and how to re-treat patients in whom VS previously failed. The fourth step was to propose management options in patients who experienced adverse reaction following previous VS.

For evaluating success or failure of VS the patient's satisfaction and the patient acceptable symptom state (PASS) ${ }^{35}$ were rated as the most useful tools in clinical practice. The patient's opinion being the primary outcome, the task force emphasized the importance of the patient's satisfaction with respect to the treatment, regardless of the results of PASS. There was a strong level of consensus to recommend re-treat in patients in whom pain occurs again and not to re-treat systematically symptom free or minimally symptomatic patients. Furthermore, consensus was obtained on recommending adapting the frequency of treatment to patients' individual situation. Young age, early-stage OA, risks factors of rapid progression and professional sportsmen were identified as clinical situations that can potentially justify re-treating patients earlier. Contra-indications to certain drugs or surgery, due to severe co-morbidities, were also considered as arguments in favour of an earlier re-treatment.

In patients in whom previous VS failed several reasons have obtained a full consensus: wrong clinical diagnosis of the source of pain (i.e. aseptic osteonecrosis, meniscal lesion, tendinopathy, subchondral bone micro-cracks, chondrocalcinosis), inappropriate protocol or inaccurate IA injection, obesity and very advanced OA (i.e. radiographic Kellgren-Lawrence grade IV). The experts consensually agreed that to improve accuracy of IA injection in the knee, the lateral midpatellar route of injection has to be preferred to anterior approach and imaging guidance has to be preferably used in difficult cases such as obese patients. In normal weight patients imaging guidance is not mandatory. However, in normalweight subjects, imaging guidance can be used according to the physician's habits. However, although imaging techniques are not necessarily 
Table 1. Statements on viscosupplementation use that obtained a unanimous or strong level of consensus. ${ }^{21}$

\begin{tabular}{|c|c|}
\hline Statements on viscosupplementation & Level of consensus \\
\hline VS is an effective treatment for mild to moderate knee OA & Unanimous \\
\hline VS is not an alternative to surgery in advanced hip OA & Unanimous \\
\hline VS is a well tolerated treatment of knee and other joints OA & Unanimous \\
\hline $\begin{array}{l}\text { Owing to its safety profile, VS should not be used only in patients who have failed } \\
\text { to respond adequately to analgesics and NSAIDs }\end{array}$ & Unanimous \\
\hline $\begin{array}{l}\text { Viscosupplementation is a 'positive' indication but not a 'lack of anything better' } \\
\text { indication }\end{array}$ & Unanimous \\
\hline The dosing regimen must be supported by evidence based medicine & Unanimous \\
\hline $\begin{array}{l}\text { Cross-linking is a proven means for prolonging intra-articular residence time of } \\
\text { hyyaluronic acid }\end{array}$ & Unanimous \\
\hline $\begin{array}{l}\text { The best approach to inject accurately viscosupplement into the knee joint is the } \\
\text { lateral mid-patellar one }\end{array}$ & Unanimous \\
\hline $\begin{array}{l}\text { When VS is performed under fluoroscopy, the amount of radiopaque contrast } \\
\text { agent must be as low as possible to avoid viscosupplement dilution }\end{array}$ & Unanimous \\
\hline VS may also be helpful in advanced stages of knee OA & Strong \\
\hline VS, when administered at early stages of OA, may have a chondroprotective effect & Strong \\
\hline Physician education influences the success of VS treatment & Strong \\
\hline $\begin{array}{l}\text { Because viscosupplements differ widely from each other, results of clinical trials } \\
\text { with a particular VS cannot be extrapolated to others }\end{array}$ & Strong \\
\hline $\begin{array}{l}\text { A single-injection regimen must be performed with products specifically } \\
\text { developed for this, whatever the joint }\end{array}$ & Strong \\
\hline Predictive factors of response to VS are poorly known and remain to be studied & Strong \\
\hline VS is a cost effective treatment for knee OA & Strong \\
\hline
\end{tabular}

NSAID, non-steroidal anti-inflammatory drug; OA, osteoarthritis; VS, viscosupplementation.

recommended, they are not contraindicated in any situation of VS. In the case of a previous nonserious local adverse reaction to VS, the experts recommended to change the viscosupplement (i.e. bacterial instead of animal origin) and/or the injection protocol. No consensus was obtained on the proposal of adding CS to HA.

EUROVISCO guidelines for the design and conduct of clinical trials assessing the diseasemodifying effect of knee VS ${ }^{32}$

The goal of this consensus driven expert meeting was to provide guidelines for the design and conduct of clinical trials assessing the disease-modifying effect of VS in knee OA.
The mechanisms of action of HA on joint tissues (synovium, cartilage, subchondral bone) are very complex and not fully understood. However, there is increasing data suggesting HA might have disease-modifying properties. ${ }^{27}$ However, human trials evidencing a clinically relevant efficacy of VS to slow down articular cartilage breakdown are still lacking.

Thirty recommendations were made regarding adequate study population, imaging and clinical tools, and soluble biomarkers assessing the joint tissue metabolism. Unanimous agreement was reached on both the need for a randomized, double-blind trial and for combining imaging data and soluble biomarker assays. 
Among the 30 recommendations only seven obtained both a strong and unanimous agreement: (1) to ensure true double-blind study design, we recommend that the injector is not the evaluator as the difference of viscosity between saline and HA can be easily identified. (2) We recommend that either cartilage changes on magnetic resonance imaging (MRI) or joint space narrowing progression on standard X-rays be the primary outcome variable in evaluating the structure-modifying effect. (3) We recommend a time interval of 1 year between two consecutive X-rays. (4) In knee OA, we recommend that $\mathrm{X}$-rays be standardized to standing postero-anterior view, Lyon-schuss or semi-flexed view, lateral view and skyline view of the patella. (5) We recommend that all X-rays be performed using a standardized procedure (patient positioning, X-ray beam distance) and evaluated centrally by a single observer. (6) We did not recommend the use of ultrasonography, computed tomography (CT) scan and CT arthrography as tools for OA diagnosis or to assess progression over time. (7) To demonstrate the disease-modifying effect of VS we recommend a combination of imaging and biological outcome measures. A decrease of soluble biomarkers of cartilage degradation over time alone does not prove the chondroprotective effect of the treatment if this effect is not complemented by the imaging examinations.

Several other recommendations obtained a high level of agreement and consensus. In multicentre studies, the task force recommended that all trial sites must comply with a standardized MRI protocol using MRI with a 3.0 Tesla (T) field strength, two-dimensional fast spin-echo sequences with intermediate-weighted and/or T2-weighted contrast with fat suppression or short tau inversion recovery. To warrant reproducible evaluation of cartilage changes over time, the experts favour semi-quantitative scoring systems over quantitative ones, as these still need further evaluation. For clinical evaluation a combination of validated outcome measures was recommended (i.e. pain on 10 point rating scale and/or WOMAC score and/or KOOS score and/or patient global assessment on 10 point rating scale and/or OMERACT-OARSI response criterion and/or PASS and/or MCII). ${ }^{35-37}$

\section{EUROVISCO recommendations for optimizing the clinical results of VS in $O A^{33}$}

The primary aim of this work was to vote on the appropriateness of the VS use in several frequent daily clinical situations and to identify phenotypes of patients who can benefit the most from VS. The task force also provided recommendations for optimizing the clinical results of VS. There was a large agreement for using VS to treat patients with mild to moderate knee and hip OA, with normal weight or moderate overweight, in whom pain is insufficiently relieved by first line therapies or who do not wish to take or have contra-indications to pain killers. The experts' opinion was in accordance with the German guidelines for the management of knee OA, ${ }^{38}$ which stipulate that subjects with contra-indications to NSAIDs/analgesics should avoid oral medications and opt for IA-HA or CSs. The working group stressed that the patient's decision remains the key element in therapeutic decision-making. For example, in very severe OA requiring surgery, VS can be performed if the patient requests it for postponement of the arthroplasty, provided that he (she) has been well informed of the risk/benefit ratio.

Obesity, severe anatomical joint involvement, large synovial fluid effusion, severe patello-femoral OA, gross joint instability and major malalignment were considered by most of the members as the main predictors of VS failure. Therefore, a good indication based on both an accurate analysis of symptoms and a careful clinical examination must be determined to improve the chances of success of VS. A good technique of injection and/or the use of an imaging guidance may enhance the chances of success of VS. Thus, for optimizing the chances of success of VS, the lateral mid-patellar approach was recommended for knee injection whilst imaging guidance was unanimously recommended for hip and ankle injection. Issues, recommendations and appropriateness for VS use that obtained a unanimous level of consensus are given in Table 2.

\section{Conclusion}

The EUROVISCO group, made up of a multidisciplinary panel of European doctors specializing in the management of OA, proposed four sets of recommendations on VS of the knee, hip and other joints. Based on the exhaustive analysis of the available literature and their own clinical experience, the experts offer a wide range of recommendations intended to help practitioners, particularly in certain cases where the specific characteristics of the patients make the therapeutic decision difficult. Among the statements that obtained a consensual agreement, the working group stressed that VS is effective in mild and moderate knee OA 
Table 2. Issues, recommendations and appropriateness for viscosupplementation use that obtained a unanimous level of consensus. ${ }^{24}$

Issues
A good indication, based on both an accurate analysis of signs, symptoms and
clinical history and a careful clinical examination may improve the chances of
success of VS.
A good indication based on a precise analysis of the radiological features may
improve the chances of success of VS.
A good technique of injection and/or the use of an imaging guidance may
enhance the chances of success of VS.
Radiological severity (KL score IV versus I-III) may influence the response of
VS in the knee.
Radiological severity (KL score IV versus I-III) may influence the response of
VS in the hip.
We recommend administering VS in the knee through a lateral patello-
femoral route.
We recommend performing VS under fluoroscopy or ultrasound guidance in
the hip.
We recommend performing VS under fluoroscopy or ultrasound guidance in
the ankle.
Patients with symptomatic, mild to moderate knee OA (JSN grade 0-2, KL
I-III), with normal weight or moderate overweight (BMI <30), not sufficiently
improved by non-pharmacological interventions and analgesics/NSAIDs.
Patients with symptomatic, mild to moderate knee OA (JSN grade 0-2, KL
I-III), with normal weight or moderate overweight (BMI <30), with contra-
indication to analgesics/NSAIDs.
using VS in daily practice

BMI, body mass index; JSN, joint space narrowing; KL, Kellgren-Lawrence; NSAID, non-steroidal anti-inflammatory drug; $\mathrm{OA}$, osteoarthritis; VS, viscosupplementation.

but is not a viable alternative to surgery in advanced OA, though it could be useful to help relieve pain in patients who cannot undergo arthroplasty. The choice of the viscosupplement and the dosing regimen must be supported by clinical trials, since HA products widely vary between themselves. Among the key recommendations for IA-HA retreatment, the experts recommended to re-treat systematically every year patients with high risk of OA progression, even if not symptomatic. For the others, re-treatment must be discussed as soon as pain reaches the PASS threshold. Among the ways for optimizing the VS outcomes, the working group underlined that a good indication, based on both an accurate analysis of radiological features and symptoms and a careful clinical examination, may improve the chances of success of VS. As well, a good technique of injection and/or the use of imaging guidance may enhance the chances of success of VS.

\section{Conflict of interest statement}

Yves Henrotin: Received honorarium from Menarini, Flexion therapeutics, IBSA, BioIberica, Expansciences, Royal canin, MagPharm, Pfizer, Fidia and LABRHA and Tilman SA, for consultant services. Founder and shareholder of KIOmed pharma. Founder and shareholder of Artialis SA.

Raghu Raman: Received honorarium from Sanofi and LABRHA for consultant services.

Pascal Richette: Received fees from Biolbérica, Fidia, IBSA, Expanscience, Genévrier, Sanofi, Rottapharm, Servier, Flexion Therapics and Ménarini.

Hervé Bard: Received speaker and expert fees from Sanofi, Pfizer, Labrha, Expanscience, TRB Chemedica

Jörg Jerosch: Received honorarium from Sanofi for speaker services

Thierry Conrozier: Received honorarium from Sanofi, MEDAC, Fidia and Labrha for expert and consultant services

Xavier Chevalier: Received fees as a Genevrier Board member, Sanofi-Aventis expert, member of the IBSA foundation, speaker in IBSA meetings, Moebius and Flexion therapics consultant

Alberto Migliore: Received consulting fees from Abbvie, BMS, MSD, Fidia, Sanofi, IBSA, Pfizer 
and Labrha, for national and international studies and courses.

Jordy Monfort: Received consulting fees from Sanofi and Bioiberica

Dominique Baron: Received speaker fees from LCA and Expansciences

\section{Funding}

This research received no specific grant from any funding agency in the public, commercial, or notfor-profit sectors.

\section{Ethics statement}

Patient involvement: This review did not require an ethics board approval because patients were not involved in the design or conduct or reporting or dissemination plans of this research. Patient consent for publication: Not required.

\section{ORCID iDs}

Thierry Conrozier (iD https://orcid.org/00000003-0353-6292

Alberto Migliore (iD https://orcid.org/0000-00027631-890X

\section{References}

1. Balazs EA and Denlinger JL. Viscosupplementation: a new concept in the treatment of osteoarthritis. F Rheumatol Suppl 1993; 39: 3-9.

2. Zhang W, Doherty M, Arden N, et al. EULAR Standing Committee for International Clinical Studies Including Therapeutics (ESCISIT). EULAR evidence based recommendations for the management of hip osteoarthritis: report of a task force of the EULAR Standing Committee for International Clinical Studies Including Therapeutics (ESCISIT). Ann Rheum Dis 2005; 64: 669-681.

3. Bruyère $\mathrm{O}$, Honvo $\mathrm{G}$, Veronese $\mathrm{N}$, et al. An updated algorithm recommendation for the management of knee osteoarthritis from the European Society for Clinical and Economic Aspects of Osteoporosis, Osteoarthritis and Musculoskeletal Diseases (ESCEO). Semin Arthritis Rheum 2019; 49: 337-350.

4. Bannuru RR, Osani MC, Vaysbrot EE, et al. OARSI guidelines for the non-surgical management of knee, hip, and polyarticular osteoarthritis. Osteoarthritis Cartilage 2019; 27: 1578-1589.
5. Steinmeyer J, Bock F, Stöve J, et al. Pharmacological treatment of knee osteoarthritis: special considerations of the new German guideline. Orthop Rev (Pavia) 2018; 10: 7782.

6. Sellam J, Courties A, Eymard F, et al. Recommendations of the French Society of Rheumatology on pharmacological treatment of knee osteoarthritis. Foint Bone Spine 2020; 87: 548-555.

7. Abdulla A, Adams N, Bone M, et al; British Geriatric Society. Guidance on the management of pain in older people. Age Ageing 2013; 42(Suppl. 1): i1-i57.

8. Trojian TH, Concoff AL, Joy SM, et al. AMSSM scientific statement concerning viscosupplementation injections for knee osteoarthritis: importance for individual patient outcomes. Br F Sports Med 2016; 50: 84-92.

9. Newberry SJ, Fitzgerald JD, Maglione MA, et al. Systematic review for effectiveness of hyaluronic acid in the treatment of severe Degenerative foint Disease (DFD) of the knee [Internet]. Rockville, MD: Agency for Healthcare Research and Quality (US), 2015.

10. Strand V, McIntyre LF, Beach WR, et al. Safety and efficacy of US-approved viscosupplements for knee osteoarthritis: a systematic review and meta-analysis of randomized, saline-controlled trials. F Pain Res 2015; 8: 217-228.

11. Bannuru RR, Schmid CH, Kent DM, et al. Comparative effectiveness of pharmacologic interventions for knee osteoarthritis: a systematic review and network meta-analysis. Ann Intern Med 2015; 162: 46-54.

12. Richette $\mathrm{P}$, Chevalier X, Ea HK, et al. Hyaluronan for knee osteoarthritis: an updated meta-analysis of trials with low risk of bias. $R M D$ Open 2015; 1: e000071.

13. Xing D, Wang B, Liu Q, et al. Intra-articular hyaluronic acid in treating knee osteoarthritis: a PRISMA- compliant systematic review of overlapping meta-analysis. Sci Rep 2016; 6: 32790.

14. Bannuru RR, Natov NS, Obadan IE, et al. Therapeutic trajectory of hyaluronic acid versus corticosteroids in the treatment of knee osteoarthritis: a systematic review and metaanalysis. Arthritis Rheum 2009; 61: 1704-1711.

15. He WW, Kuang MJ, Zhao J, et al. Efficacy and safety of intraarticular hyaluronic acid and corticosteroid for knee osteoarthritis: a metaanalysis. Int $\mathcal{F}$ Surg 2017; 39: 95-103.

16. Chevalier X, Sheehan B, Whittington C, et al. Efficacy and safety of hylan G-F 20 versus 
intra-articular corticosteroids in people with knee osteoarthritis: a systematic review and network meta-analysis. Clin Med Insights Arthritis Musculoskelet Disord 2020; 13 : 1179544120967370.

17. O'Hanlon CE, Newberry SJ, Booth M, et al. Hyaluronic acid injection therapy for osteoarthritis of the knee: concordant efficacy and conflicting serious adverse events in two systematic reviews. Syst Rev 2016; 5: 186.

18. Altman R, Lim S, Steen RG, et al. Hyaluronic acid injections are associated with delay of total knee replacement surgery in patients with knee osteoarthritis: evidence from a large U.S. health claims database. PLoS One 2015; 10: e0145776.

19. Altman R, Fredericson M, Bhattacharyya SK, et al. Association between hyaluronic acid injections and time-to-total knee replacement surgery. F Knee Surg 2016; 29: 564-570.

20. Etter K, Chitnis AS, Holy CE, et al. Highconcentration nonavian high-molecular weight hyaluronan injections and time-to-total knee replacement surgery. F Comp Eff Res 2020; 9: 795-805.

21. Kolasinski SL, Neogi T, Hochberg MC, et al. 2019 American College of Rheumatology/Arthritis Foundation guideline for the management of osteoarthritis of the hand, hip, and knee. Arthritis Care Res (Hoboken) 2020; 72: 149-162.

22. Jevsevar DS. Treatment of osteoarthritis of the knee: evidence-based guideline, 2 nd edition. F Am Acad Orthop Surg 2013; 21: 571-576.

23. Jevsevar DS, Shores PB, Mullen K, et al. Mixed treatment comparisons for nonsurgical treatment of knee osteoarthritis: a network meta-analysis. f Am Acad Orthop Surg 2018; 26: 325-336.

24. Meiyappan KP, Cote MP, Bozic KJ, et al. Adherence to the American Academy of Orthopaedic Surgeons clinical practice guidelines for nonoperative management of knee osteoarthritis. F Arthroplasty 2020; 35: 347-352.

25. Carlson VR, Ong AC, Orozco FR, et al. Compliance with the AAOS guidelines for treatment of osteoarthritis of the knee: a survey of the American Association of Hip and Knee Surgeons. F Am Acad Orthop Surg 2018; 26: 103-107.

26. Printz JO, Lee JJ, Knesek M, et al. Conflict of interest in the assessment of hyaluronic acid injections for osteoarthritis of the knee: an updated systematic review. F Arthroplasty 2013; 28(Suppl. 8): 30-33.e1.

Visit SAGE journals online journals.sagepub.com/ home/tab

(SAGE journals
27. Nicholls MA, Fierlinger A, Niazi F, et al. The disease-modifying effects of hyaluronan in the osteoarthritic disease state. Clin Med
Insights Arthritis Musculoskelet Disord 2017; 10: 1179544117723611.

28. Altman RD, Manjoo A, Fierlinger A, et al. The mechanism of action for hyaluronic acid treatment in the osteoarthritic knee: a systematic review. BMC Musculoskelet Disord 2015; 16: 321.

29. Bhadra AK, Altman R, Dasa V, et al. Appropriate use criteria for hyaluronic acid in the treatment of knee osteoarthritis in the United States. Cartilage 2017; 8: 234-254.

30. Henrotin Y, Raman R, Richette P, et al. Consensus statement on viscosupplementation with hyaluronic acid for the management of osteoarthritis. Semin Arthritis Rheum 2015; 45: 140-149.

31. Raman R, Henrotin Y, Chevalier X, et al. Decision algorithms for the retreatment with viscosupplementation in patients suffering from knee osteoarthritis: recommendations from the EUROpean VIScosupplementation COnsensus Group (EUROVISCO). Cartilage 2018; 9: 263-275.

32. Henrotin Y, Chevalier X, Raman R, et al. EUROVISCO guidelines for the design and conduct of clinical trials assessing the diseasemodifying effect of knee viscosupplementation. Cartilage 2020: 11: 60-70.

33. Conrozier T, Monfort J, Chevalier X, et al. EUROVISCO recommendations for optimizing the clinical results of viscosupplementation in osteoarthritis. Cartilage 2020: 11: 47-59.

34. Altman R, Hackel J, Niazi F, et al. Efficacy and safety of repeated courses of hyaluronic acid injections for knee osteoarthritis: a systematic review. Semin Arthritis Rheum 2018; 48: 168-175.

35. Tubach F, Ravaud P, Martin-Mola E, et al. Minimum clinically important improvement and patient acceptable symptom state in pain and function in rheumatoid arthritis, ankylosing spondylitis, chronic back pain, hand osteoarthritis, and hip and knee osteoarthritis: results from a prospective multinational study. Arthritis Care Res 2012; 64: 1699-1707.

36. Roos EM, Roos HP, Lohmander LS, et al. Knee Injury and Osteoarthritis Outcome Score [KOOS]-development of a self-administered outcome measure. F Orthop Sports Phys Ther 1998; 28: 88-96.

37. Pham T, Van Der Heijde D, Lassere M, et al. Outcome variables for osteoarthritis clinical trials: the OMERACT-OARSI set of responder criteria. F Rheumatol 2003; 30: 1648-1654.

38. Steinmeyer J, Bock F, Stöve J, et al. Pharmacological treatment of knee osteoarthritis: special considerations of the new German guideline. Orthop Rev (Pavia) 2018; 10: 7782. 